\title{
Spontaneous rupture of abdominal wall hernia
}

\author{
Takanobu Hirosawa ${ }^{1}$ and Taro Shimizu ${ }^{2}$ \\ ${ }^{1}$ Dokkyo Medical University \\ ${ }^{2}$ Dokkyo Medical University Hospital
}

December 6, 2021

Spontaneous rupture of abdominal wall hernia

Takanobu Hirosawa ${ }^{1}$ MD, Taro Shimizu ${ }^{1}$ MD, PhD, MPH, MBA

1. Department of Diagnostic and Generalist Medicine, Dokkyo Medical University

All authors have no conflict of interest and grant to declare.

Written informed consent was obtained from the patient to publish this report in accordance with the journal's patient consent policy.

\section{Takanobu Hirosawa, MD}

Corresponding author

Department of Diagnostic and Generalist Medicine, Dokkyo Medical University, Tochigi, Japan

880 Kitakobayashi, Mibu-cho, Simotsuga-gun Tochigi 321-0293, Japan

TEL: +81-282-86-1111 FAX:+81-282-86-4775

e-mail:t.hirosawa1983@gmail.com

Key terms: abdominal wall hernia, spontaneous rupture

Key Clinical Message

Crepitus on palpation of abdominal wall is a sign of the presence of gas within the subcutaneous tissue (1). In this case, careful inspection and palpation of the abdomen are concluded to detect the spontaneous rupture of abdominal wall hernia.

Question

What is an important consideration in the crepitus on palpation of the abdominal wall?

Answer

Spontaneous rupture of the abdominal wall is important to consider even without trauma.

Case Description

A 70-year-old bed-ridden man was transferred to this hospital due to fever and wet-cough. His past medical history included embolic cerebral infarction and spinal cord injury. The initial physical examination revealed crackles in his chest. Also, there was a bulging of the skin on his right lower abdominal wall with ecchymosis on the top (Figure 1A). The bulging was reducible manually at that time. Further questioning to his family member revealed that he had no recent abdominal trauma, vomiting, coughing, or constipation. He was admitted under the tentative diagnosis of pneumonia, given the symptom and physical finding. After the 
admission, his symptoms improved with intravenous antibiotics therapy. However, his right lower abdominal wall was found to be remarkably swollen (Figure 1B) and crepitus was felt on palpation. An abdominal CT without contrast showed abdominal wall and colon rupture (Figure 2). Emergent surgery for the ruptured abdominal wall hernia was offered, but his family declined further intervention because of his poor general status. He passed away soon after. Autopsy revealed colon rupture to the abdominal wall without neoplastic lesion or inflammation. The lesion was finally attributed to the spontaneous rupture of abdominal wall hernia communicating to the subcutaneous tissue.

Author's contribution

TH wrote the case report. TS supervised it. Both authors approved the final version.

\section{References}

Charles M. Ferguson. (1990). Clinical Methods: The History, Physical, and Laboratory Examinations. 3rd edition. Boston: Butterworths. Chapter 93.Inspection, Auscultation, Palpation, and Percussion of the Abdomen.

Figure legend

Figure 1. A bulging of the right lower abdominal wall skin with ecchymosis on the top (A). The patient's abdominal wall was found to be remarkably swollen (B).

Figure 2. Abdominal CT without contrast showed abdominal wall and colon rupture.

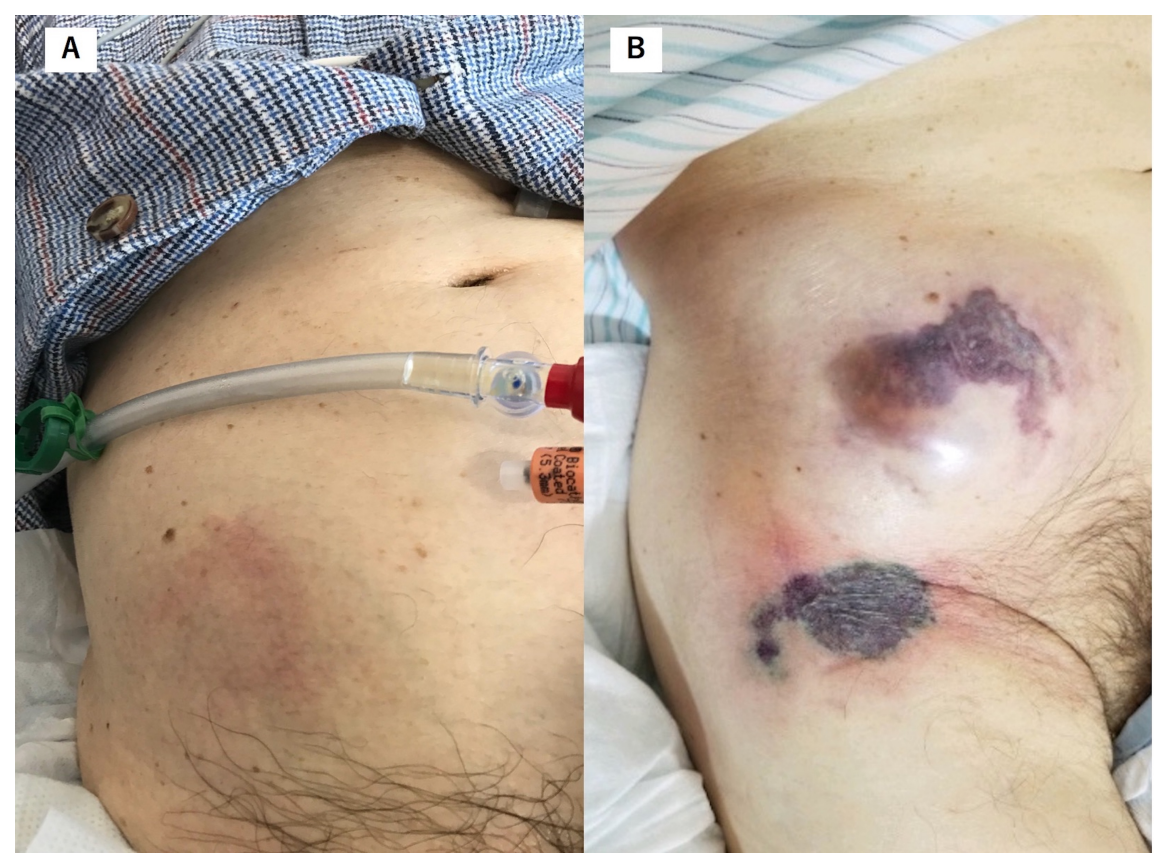




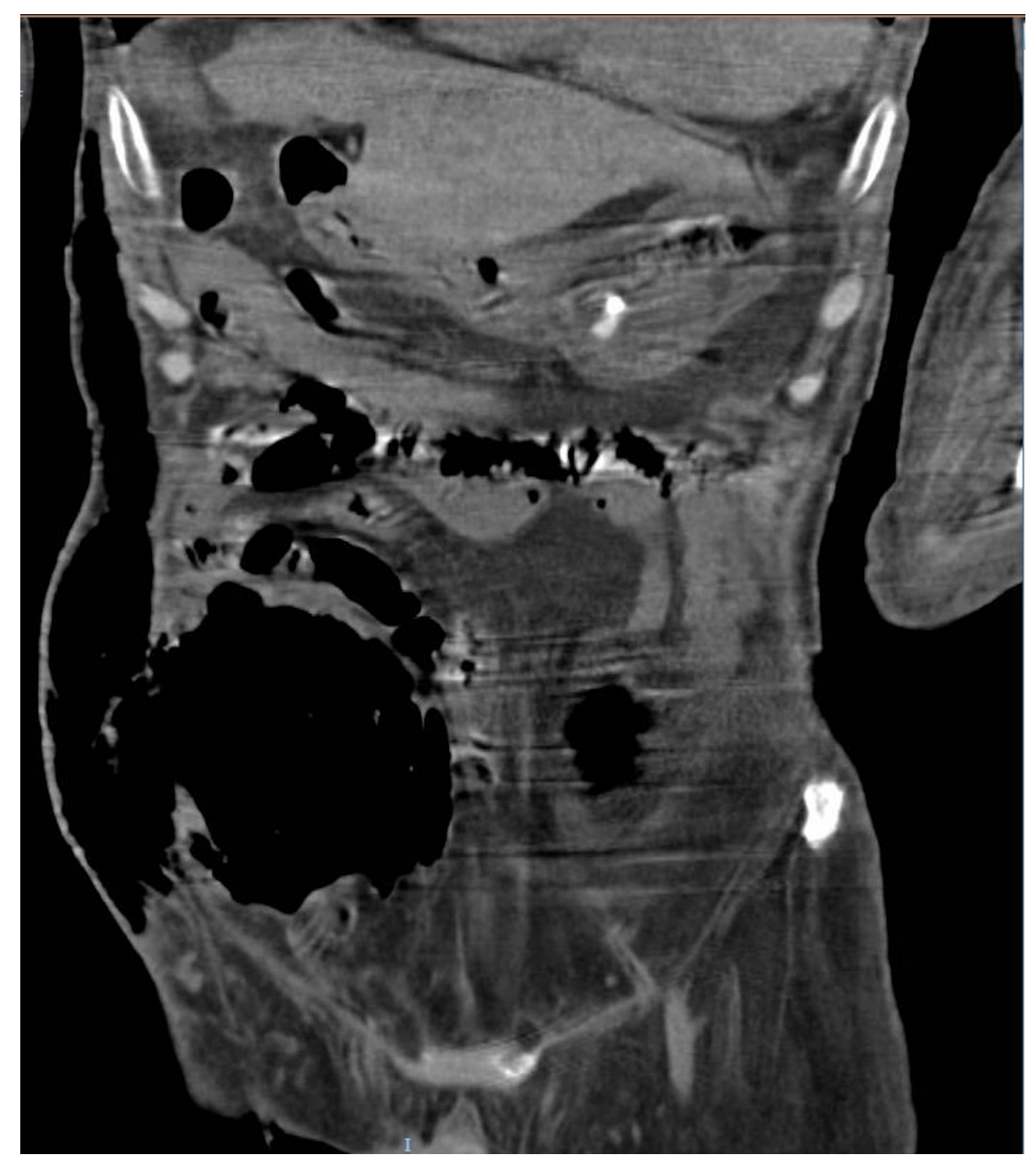

